\title{
Study on Amplitude Modulation Principle of Chaotic System
}

\author{
Jian-Rong Du, Chun-Lai Li $\mathbb{D}$, Kun Qian, Zhao-Yu Li, and Wen Li \\ College of Physics and Electronics, Hunan Institute of Science and Technology, Yueyang 414006, China \\ Correspondence should be addressed to Chun-Lai Li; hnistlichl@163.com
}

Received 2 July 2019; Revised 16 November 2019; Accepted 3 December 2019; Published 11 February 2020

Guest Editor: Mahendra K. Gupta

Copyright (C) 2020 Jian-Rong Du et al. This is an open access article distributed under the Creative Commons Attribution License, which permits unrestricted use, distribution, and reproduction in any medium, provided the original work is properly cited.

Exploring the amplitude modulation phenomenon of chaotic signal has become a subject of great concern in recent years. This paper mainly concentrates on the preliminary study on amplitude modulation principle of a chaotic system. First, two 3D chaotic systems with quadratic product terms are introduced for studying the amplitude modulation phenomenon of chaotic signal. It is found that the signal amplitude of the first system can be controlled by partial quadratic coefficient. But for the second system, none of nonlinear coefficient can be employed to control the signal amplitude. Then, the amplitude modulation principle of chaotic system is preliminarily studied by exploring the intrinsic relationship between nonzero equilibrium point and phase space trajectory, and it is further validated by introducing unified parameter to the two 3D chaotic systems. As a necessary condition, the principle provides a feasible and simple method for constructing and analyzing an amplitude modulation chaotic system.

\section{Introduction}

Chaos has evoked much attention in many scientific fields due to its unique characteristics, such as sensitivity to initial conditions and parameter deviations, strange attractor with locally unbounded but globally bounded trajectory, unpredictability of future behavior, and so on [1-4]. In the past few decades, the issue of construction, analysis, and application of chaotic systems has become a very active topic [5-10].

The signal amplitude of a chaotic system can be often modulated by controlling one or more coefficients in the dynamical equations, while the Lyapunov exponents and power spectral density remain invariable [11-14]. Since the variables can be rescaled by directly controlling the amplitude parameter to avoid the exceeding limitations of bandwidth and amplitude, this kind of system can resolve the contradiction of signal processing and information retention in practical amplification circuit, so it is a promising type of system for the reality of chaotic encryption, chaotic radar, chaotic communication, and chaotic signal processing.

Generally, for the presented amplitude modulation systems with quadratic nonlinearity, the amplitude parameters are the coefficients of quadratic terms, which can nonlinearly modulate the signal amplitude of partial or total state variables [11-15]. In fact, while chaotic system holds the only nonlinear term, the corresponding coefficient can control the signal amplitude since it uniquely determines the scale of the variables [16-18]. As an example, a simple chaotic system with a single nonlinearity $x_{1}^{3}$ is recalled as $\dot{x}_{1}=x_{2}, \dot{x}_{2}=x_{3}, \dot{x}_{3}=b x_{1}-x_{2}-a x_{3}-b x_{1}^{3}$ [16]. Accordingly, it holds the resulting system $\dot{x}_{1}=x_{2}, \dot{x}_{2}=x_{3}, \dot{x}_{3}=b x_{1}-x_{2}-$ $a x_{3}-b p x_{1}^{3}$ with the substitution $x_{1} \longrightarrow \sqrt{p} x_{1}$, $x_{2} \longrightarrow \sqrt{p} x_{2}, x_{3} \longrightarrow \sqrt{p} x_{3}$. Thereby, the coefficient of the nonlinear term $x_{1}^{3}$ can control the amplitude of $x_{1}, x_{2}, x_{3}$ according to $1 / \sqrt{p}$. However, it is found in the recent work that for the dynamical system with exponential nonlinearity, the coefficient of quadratic term cannot provide amplitude modulation [19]. Thus, naturally, an interesting question is raised as "whether each coefficient of nonlinear term in chaotic system can control the signal amplitude." Another more important question is "what is the principle of constructing and analyzing amplitude modulation chaotic system." However, as far as we know, there is little information about this kind of system in the literature so far, so it still remains open and challenging.

This paper attempts to provide some answers to these questions by introducing two carefully screened chaotic systems. Differing from the system with a single linear or a 
single nonlinear term, the present systems have nonunique linear terms and three quadratic cross-product terms. Basic dynamics of these two nonlinear systems are analyzed theoretically and numerically. Somewhat surprisingly, only one coefficient of the quadratic nonlinearity in the first system can be employed to control the amplitude of chaotic signal. But for the second system, none of the coefficient of quadratic nonlinearity can be employed to control the amplitude of chaotic signal. The discovery that not all coefficients of nonlinear term can provide amplitude control is of interest and inspiration. As a further concern in this work, the amplitude modulation principle of chaotic system is addressed, based on the analysis of the intrinsic relationship between nonzero equilibrium point and phase space trajectory. Although it is not a sufficient and necessary condition for amplitude modulation, the proposed principle provides a feasible method for constructing and analyzing amplitude modulation chaotic system. Furthermore, this method is simple in actual operation and will hopefully enlighten for revealing the amplitude modulation mechanism of chaotic system.

This paper is organized as follows. Following the introduction, we propose a chaotic system with partial coefficient of nonlinearity employed to control amplitude. In
Section 3, we introduce another chaotic system with no coefficient of nonlinearity employed to control amplitude. The principle of amplitude modulation is addressed in Section 4. Finally, some concluding remarks are drawn in Section 5 .

\section{Chaotic System with Partial Coefficient of Nonlinearity for Amplitude Control}

2.1. System Description. The reported system possesses four linear terms and three quadratic cross-product terms, which is given by the following ordinary differential equations:

$$
\left\{\begin{array}{l}
\dot{x}_{1}=a\left(x_{2}-x_{1}\right)+d x_{2} x_{3}, \\
\dot{x}_{2}=b x_{2}-e x_{1} x_{3}, \\
\dot{x}_{3}=-c x_{3}+f x_{1} x_{2} .
\end{array}\right.
$$

It is easy to know that the proposed system is symmetric with respect to the $x_{3}$-axis, as shown by the coordinate transformation $\left(x_{1}, x_{2}, x_{3}\right) \longrightarrow\left(-x_{1},-x_{2}, x_{3}\right)$.

By considering the equilibrium condition $a\left(x_{2}-x_{1}\right)+$ $d x_{2} x_{3}=0, b x_{2}-e x_{1} x_{3}=0,-c x_{3}+f x_{1} x_{2}=0$, five equilibrium points of system (1) are determined as

$$
\begin{aligned}
& P_{0}(0,0,0), \\
& P_{1}\left(\sqrt{\frac{b c}{e f}}, \frac{-a \sqrt{c e}+\sqrt{c e} \sqrt{a^{2}+(4 a b d / e)}}{2 d \sqrt{b f}}, \frac{-a+\sqrt{a^{2}+(4 a b d / e)}}{2 d}\right), \\
& P_{2}\left(\sqrt{\frac{b c}{e f}}, \frac{-a \sqrt{c e}-\sqrt{c e} \sqrt{a^{2}+(4 a b d / e)}}{2 d \sqrt{b f}}, \frac{-a-\sqrt{a^{2}+(4 a b d / e)}}{2 d}\right), \\
& P_{3}\left(-\sqrt{\frac{b c}{e f}}, \frac{a \sqrt{c e}+\sqrt{c e} \sqrt{a^{2}+(4 a b d / e)}}{2 d \sqrt{b f}},-\frac{a+\sqrt{a^{2}+(4 a b d / e)}}{2 d \sqrt{b f}}\right), \\
& P_{4}\left(-\sqrt{\frac{b c}{e f}}, \frac{a \sqrt{c e}-\sqrt{c e} \sqrt{a^{2}+(4 a b d / e)}}{2 d \sqrt{b f}},-\frac{a-\sqrt{a^{2}+(4 a b d / e)}}{2 d \sqrt{b f}}\right) .
\end{aligned}
$$

And the characteristic equation is deduced as

$$
\begin{aligned}
& \varphi(\lambda)=-\lambda^{3}+(-a+b-c) \lambda^{2}+\left[a b+b c-a c+d f x_{2}^{2}-a e x_{3}-d e x_{3}^{2}-e f x_{1}^{2}\right] \lambda \\
&+\left[a b c-a e f x_{1} x_{2}-b d f x_{2}^{2}-a c e x_{3}-c d e x_{3}^{2}-a e f x_{1}^{2}\right] . \\
& P_{0}: \lambda_{1}=-28, \lambda_{2}=20, \lambda_{3}=-3, \\
& \text { parameter set } a=28, b=20, c=3, P_{1,4}: \lambda_{1}=-18.1945, \lambda_{2}=3.5973+13.8748 i, \\
& \text { four nonzero equilibrium points are } \lambda_{3}=3.5973-13.8748 i, \\
& \text { 4641, 3.0743, 3.5499), } P_{2}(3.4641, P_{2,3}: \lambda_{1}=-43.6337, \lambda_{2}=16.3169+22.2518 i, \\
& \text { (-3.4641, 27.3230,-31.5499), and } P_{4} \lambda_{3}=16.3169-22.2518 i .
\end{aligned}
$$

$(-3.4641,-3.0743,3.5499)$. And the corresponding characteristic roots are
When selecting the parameter set $a=28, b=20, c=3$, $d=1, e=5$, and $f=1$, the four nonzero equilibrium points are calculated as $P_{1}(3.4641,3.0743,3.5499), P_{2}$ (3.4641, 
Obviously, equilibrium point $P_{0}$ is a saddle-node with two-dimensional stable manifold and one-dimensional unstable manifold. But for the remaining nonzero equilibrium points $P_{1}$ to $P_{4}, \lambda_{1}$ is a negative real number and $\lambda_{2}$ and $\lambda_{3}$ become a pair of complex conjugate roots with positive real parts. Accordingly, the four equilibrium points are saddle-focus points with two-dimensional unstable manifold and one-dimensional stable manifold.

When selecting $a=28, b=20, c=3, d=1, e=5$, and $f=1$ and computing time $5000 \mathrm{~s}$, the three finite time Lyapunov exponents of system (1) are calculated by orthogonal method as $3.29613>0,0.01355,-19.34679<0$. And the KaplanYorke dimension is obtained as $D_{K Y}=2+(3.29613+$ $0.01355) / 19.34679=2.1711$, revealing a fractional feature. Therefore, system (1) is chaotic. The corresponding chaotic phase diagrams and Poincare mapping on plane $x_{2}=0$ are depicted in Figure 1.

\subsection{Analysis of Amplitude Modulation}

Theorem 1. The parameter $f$ in cross-product term $x_{1} x_{2}$ is a local parameter of nonlinear amplitude modulation, which can control the signal amplitude of $x_{1}, x_{2}$ by the power function of index $-1 / 2$ respectively, but the amplitude of variable $x_{3}$ remains in the same range; besides, the Lyapunov exponent spectrum remains unchanged with the variation of parameter $f$.

Proof. Considering the variable substitution $x_{1}=u_{1} / \sqrt{h}$, $x_{2}=u_{2} / \sqrt{h}, x_{3}=u_{3}(h>0)$, system (1) is turned to

$$
\left\{\begin{array}{l}
\dot{u}_{1}=a\left(u_{2}-u_{1}\right)+d u_{2} u_{3}, \\
\dot{u}_{2}=b u_{2}-e u_{1} u_{3}, \\
\dot{u}_{3}=-c u_{3}+(f / h) u_{1} u_{2} .
\end{array}\right.
$$

Therefore, when parameter $f$ increases linearly, the signal amplitude of system variables $x_{1}, x_{2}$ change according to the power function of index $-1 / 2$, respectively, but the amplitude of variable $x_{3}$ is in the same range.

When substituting the equilibrium point $P_{0}$ into characteristic equation (3), it holds

$$
\varphi(\lambda)=-\lambda^{3}+(-a+b-c) \lambda^{2}+(a b+b c-a c) \lambda+a b c .
$$

In equation (6), the influence of parameter $f$ is eliminated. We can draw a similar conclusion for the other equilibrium points. As an illustration, we insert the equilibrium $P_{1}$ into expression (3) obtaining

$$
\begin{aligned}
\varphi(\lambda)= & -\lambda^{3}+(-a+b-c) \lambda^{2}+\left\{a b+b c-a c+\frac{c e\left[2 a^{2}+(4 a b d / e)-2 a \sqrt{a^{2}+(4 a b d / e)}\right]}{4 b d}\right. \\
& \left.-a e \frac{-a+\sqrt{a^{2}+(4 a b d / e)}}{2 d}-\frac{2 a^{2} e+4 a b d-2 a e \sqrt{a^{2}+(4 a b d / e)}}{4 d}-b c\right\} \lambda \\
& +\left\{a b c-a e \frac{c \sqrt{b e}\left(-a+\sqrt{a^{2}+(4 a b d / e)}\right)}{2 d \sqrt{b e}}-\frac{c e\left[2 a^{2}+(4 a b d / e)-2 a \sqrt{a^{2}+(4 a b d / e)}\right]}{4 d}\right. \\
& \left.-a c e \frac{-a+\sqrt{a^{2}+(4 a b d / e)}}{2 d}-c d e \frac{2 a^{2}+(4 a b d / e)-2 a \sqrt{a^{2}+(4 a b d / e)}}{4 d^{2}}-a b c\right\} .
\end{aligned}
$$

That is, parameter $f$ does not produce effect on the characteristic root of equation (7). Therefore, when parameter $f$ varies in field of real number, the Lyapunov exponent spectrum remains constant. This completes the proof.

The corresponding bifurcation diagram and Lyapunov exponent spectrum versus $f$ are shown in Figure 2, which authenticates the theoretical results.

It is generally accepted that for the quadratic chaotic systems, the coefficients of nonlinear terms can modulate the signal amplitude of partial or total state variables [11-14]. The bifurcation diagram for $e \in[0,20]$ is shown in Figure 3(a). Superficially, the coefficient $e$ can modulate the signal amplitude nonlinearly. But from the enlarged view, one can see that there emerges a visible periodic window, and the Lyapunov exponent spectrum further verifies the observation, as depicted in Figures 3(b) and 3(c). The concrete bifurcation diagram and Lyapunov exponent spectrum versus $d$ also show that not all coefficients of quadratic terms can modulate the signal amplitude, which is illustrated in Figure 4. In spite of this, the chaos of the reported system is still robust in a large range of parameters $d$ and $e$. Consequently, the system can be recommended as an important candidate in secure communication.

\section{Chaotic System with Noncoefficient of Nonlinearity for Amplitude Control}

3.1. System Description. We consider another three-dimensional autonomous system with five linear terms and three quadratic cross-product terms, as follows: 


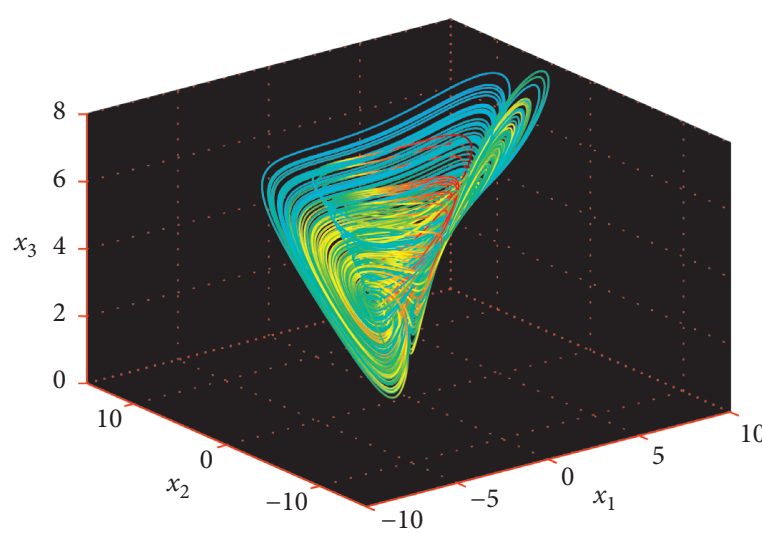

(a)

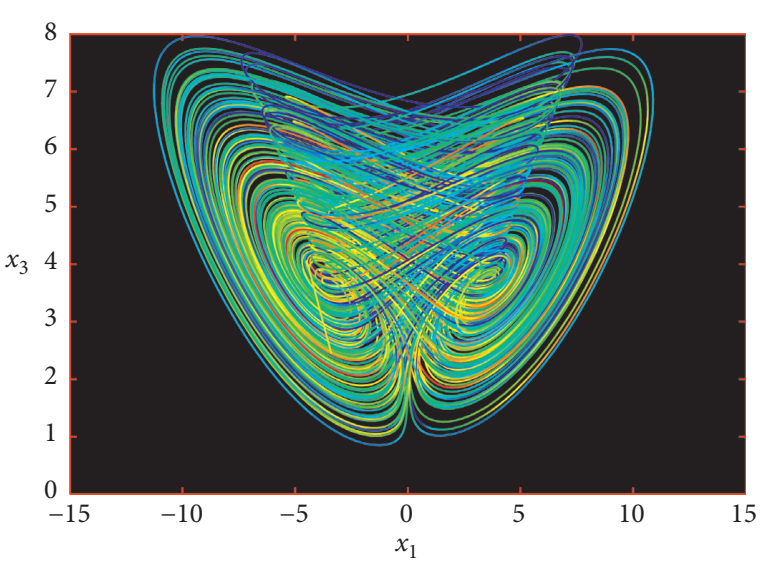

(b)

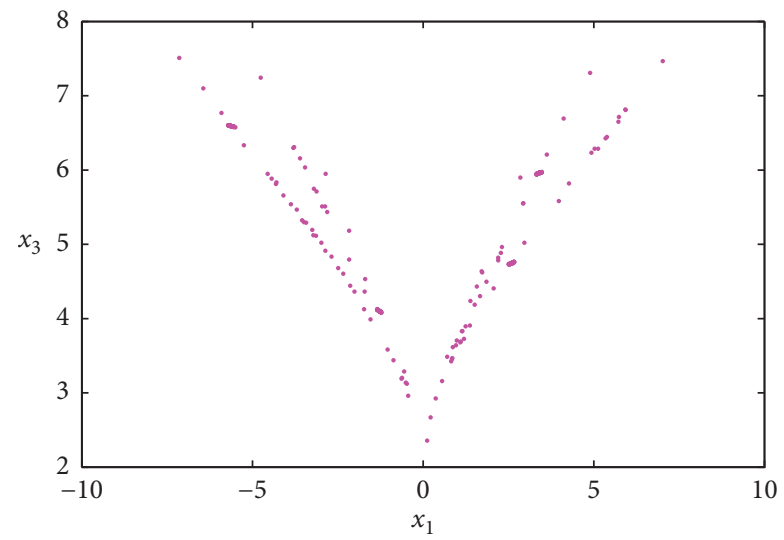

(c)

Figure 1: (a) $x_{1}-x_{2}-x_{3}$ phase portrait; (b) $x_{1}-x_{3}$ phase portrait; (c) Poincare mapping.

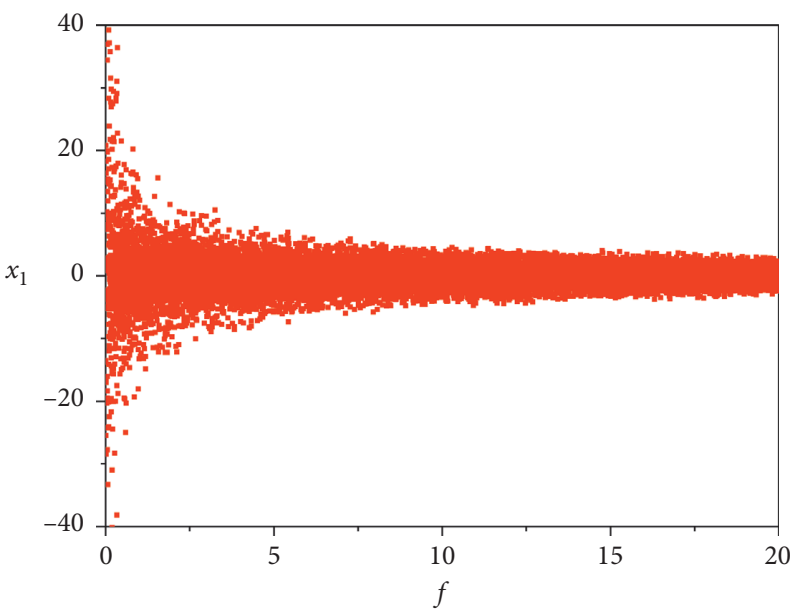

(a)

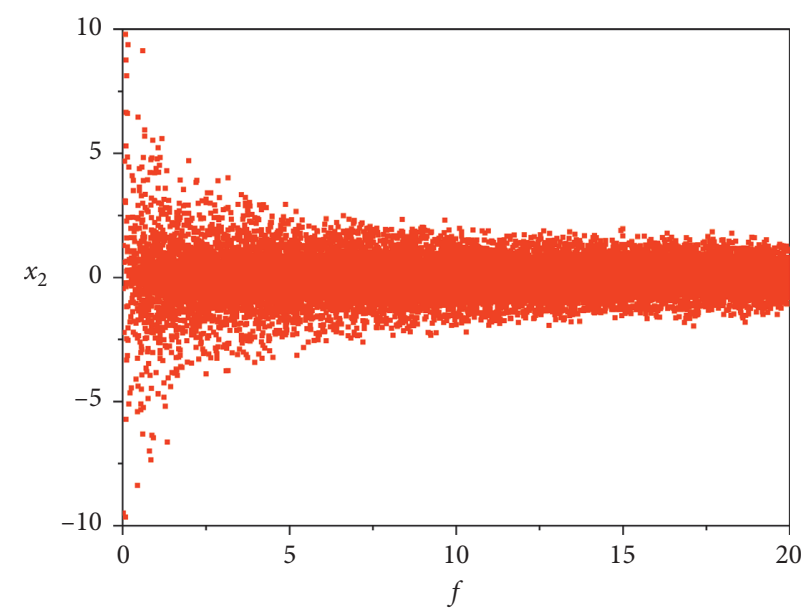

(b)

Figure 2: Continued. 


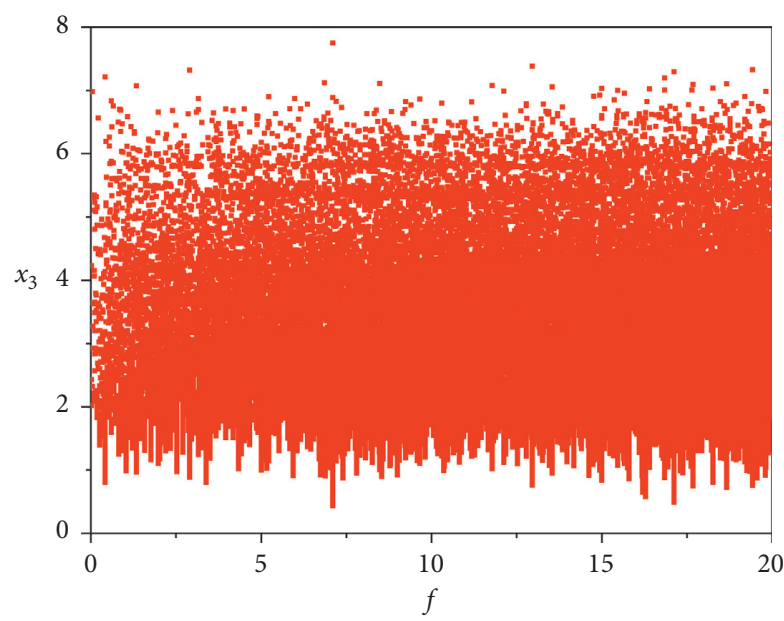

(c)

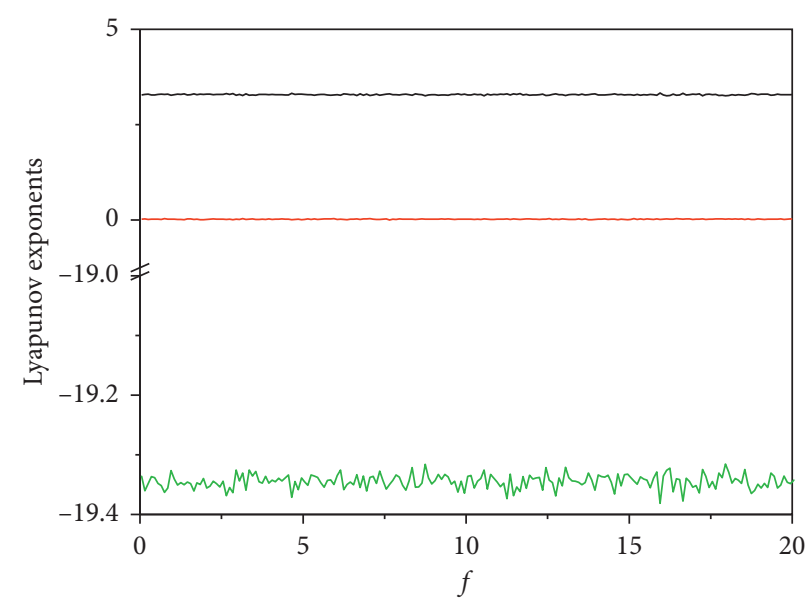

(d)

Figure 2: $(\mathrm{a}, \mathrm{b}, \mathrm{c})$ Bifurcation diagram versus $f$; (d) finite time Lyapunov exponent spectrum versus $f$.

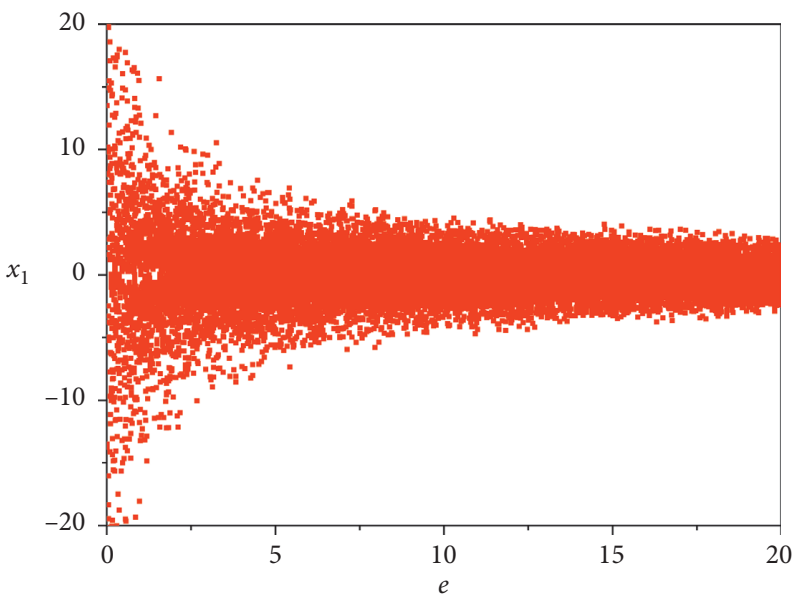

(a)

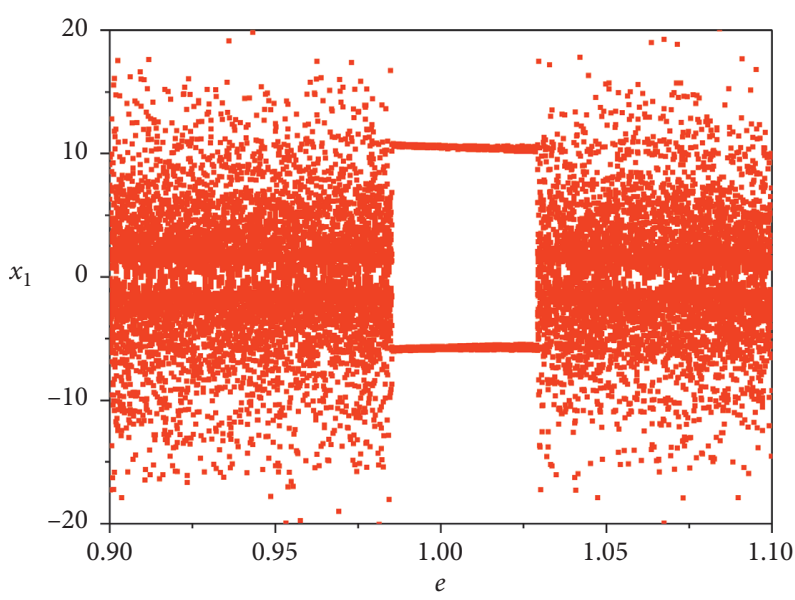

(b)

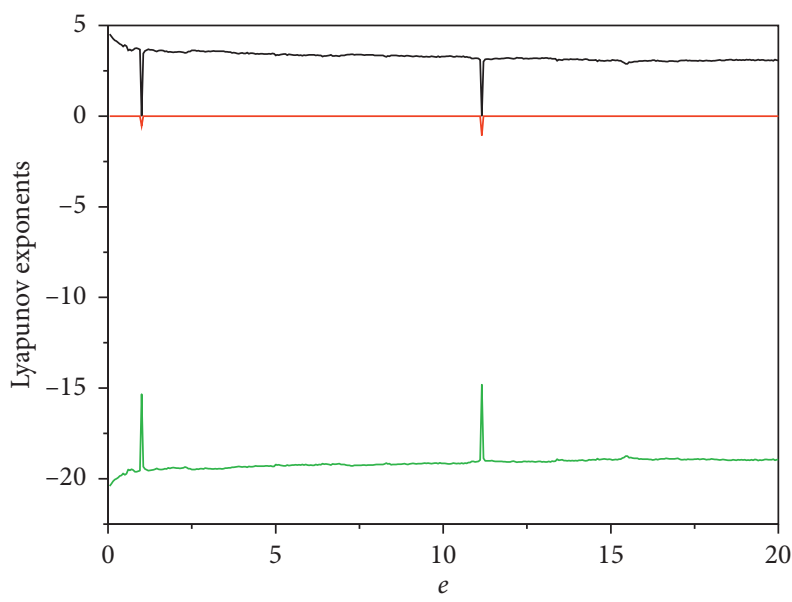

(c)

Figure 3: (a, b) Bifurcation diagram versus $e$; (c) finite time Lyapunov exponent spectrum versus $e$. 


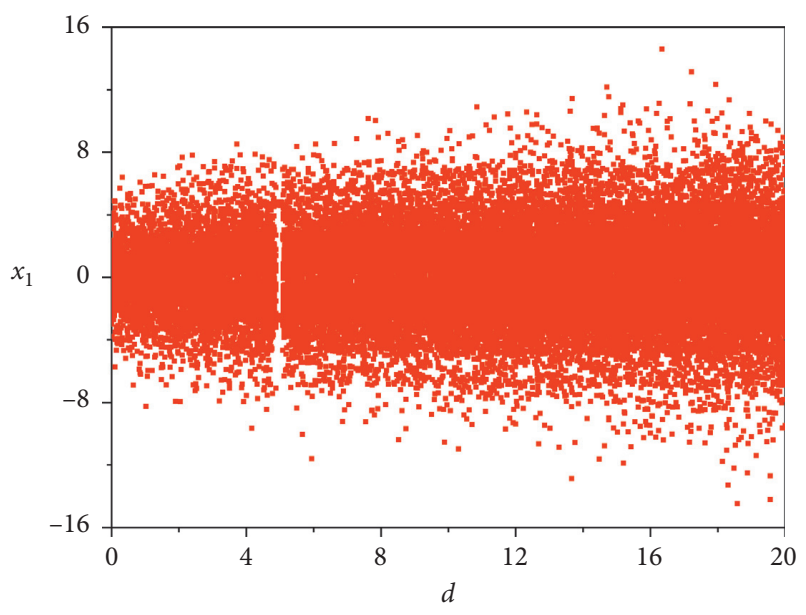

(a)

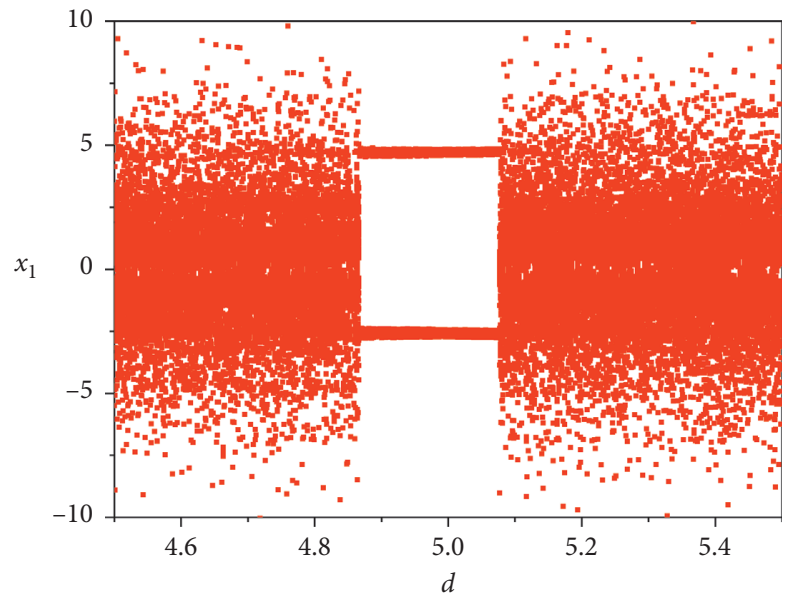

(b)

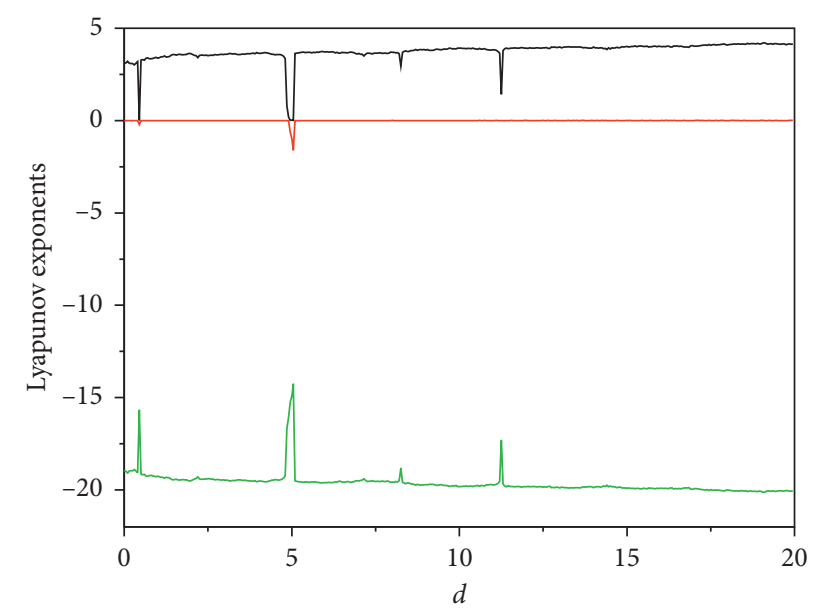

(c)

Figure 4: (a, b) Bifurcation diagram versus $d$; (c) finite time Lyapunov exponent spectrum versus $d$.

$$
\left\{\begin{array}{l}
\dot{x}_{1}=a x_{2}-g x_{1}+d x_{2} x_{3}, \\
\dot{x}_{2}=b x_{1}-e x_{1} x_{3}, \\
\dot{x}_{3}=k x_{1}-c x_{3}+f x_{1} x_{2} .
\end{array}\right.
$$

System (8) possesses three equilibrium points, which are, respectively, described by

$$
\begin{aligned}
& P_{0}(0,0,0), \\
& P_{1}\left((a e+b d) \frac{-k+\sqrt{k^{2}+(4 b c g f /(a e+b d))}}{2 g e f}, \frac{-k+\sqrt{k^{2}+(4 b c g f /(a e+b d))}}{2 f}, \frac{b}{e}\right), \\
& P_{2}\left((a e+b d) \frac{-k-\sqrt{k^{2}+(4 b c g f /(a e+b d))}}{2 g e f}, \frac{-k-\sqrt{k^{2}+(4 b c g f /(a e+b d))}}{2 f}, \frac{b}{e}\right) .
\end{aligned}
$$

When selecting the parameter set $a=24, b=12, c=1$, $d=1, e=1, f=1, k=1$, and $g=6$, the two nonzero equilibrium points are obtained as $P_{1}(6,1,12)$ and $P_{2}(-12$, $-2,12)$. The corresponding characteristic roots are 


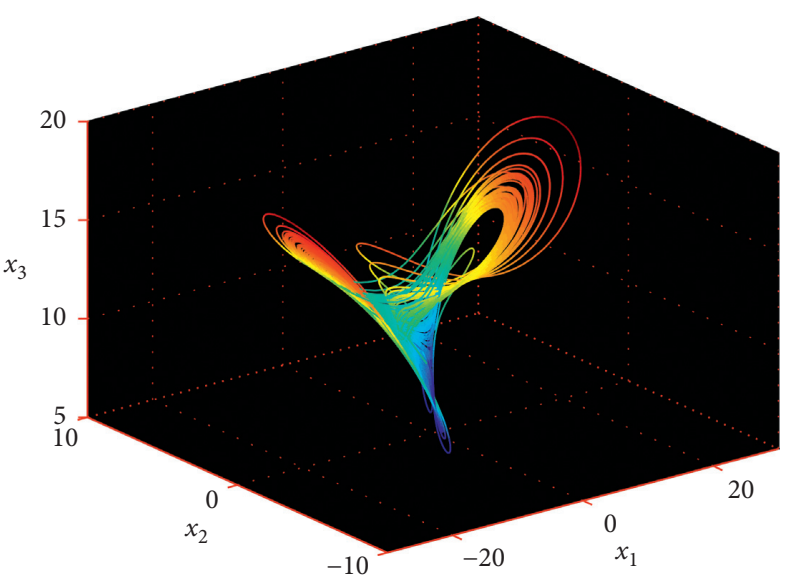

(a)

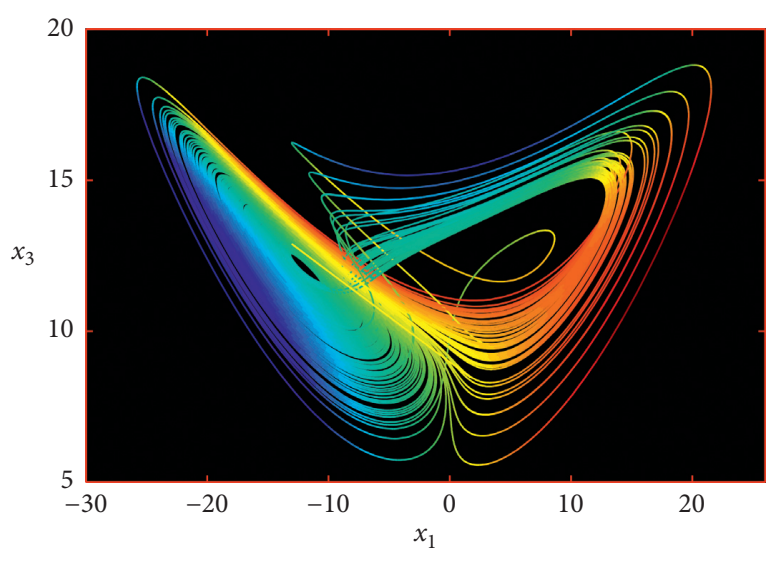

(b)

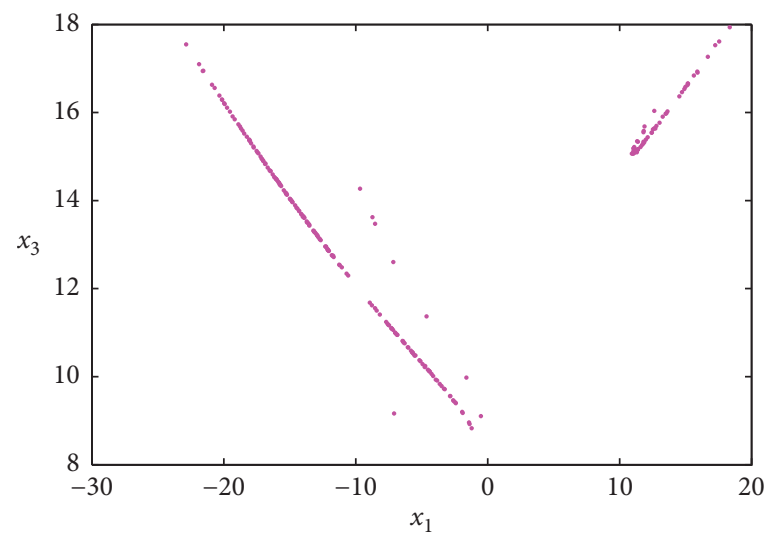

(c)

FIgURE 5: (a) $x_{1}-x_{2}-x_{3}$ phase portrait; (b) $x_{1}-x_{3}$ phase portrait; (c) Poincare mapping.

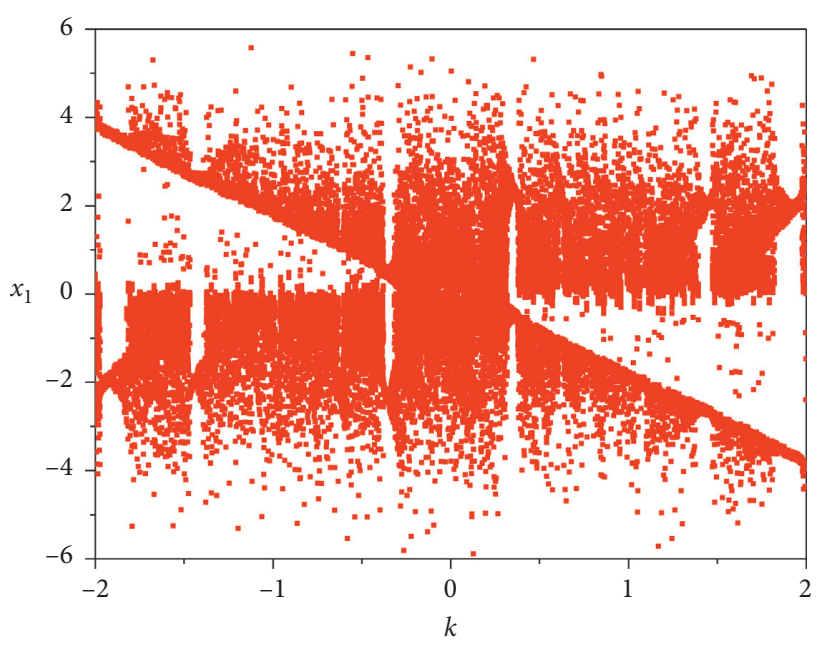

(a)

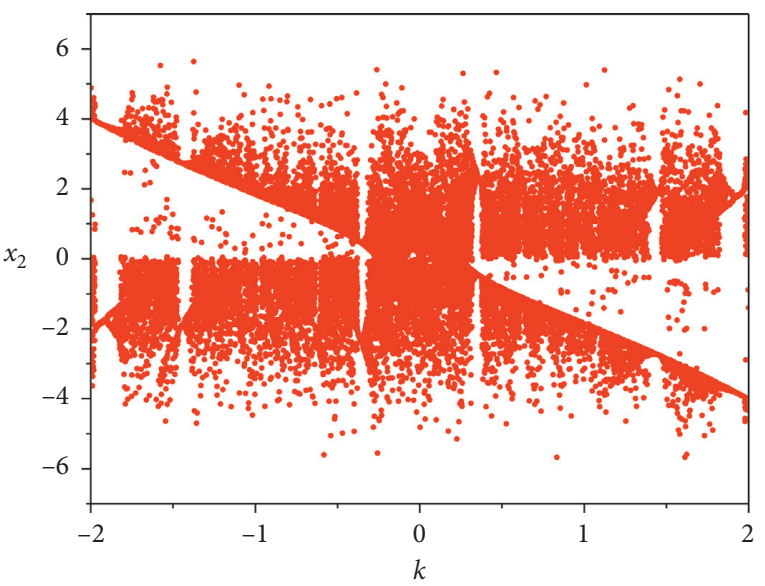

(b)

Figure 6: Continued. 


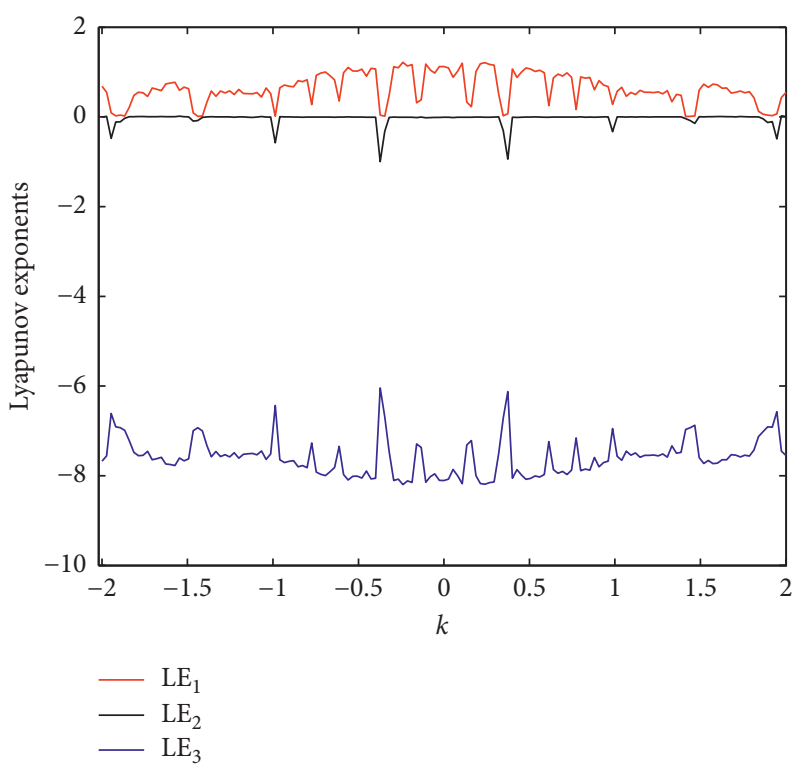

(c)

Figure 6: (a, b) Bifurcation diagram versus $k$; (c) finite time Lyapunov exponent spectrum versus $k$.

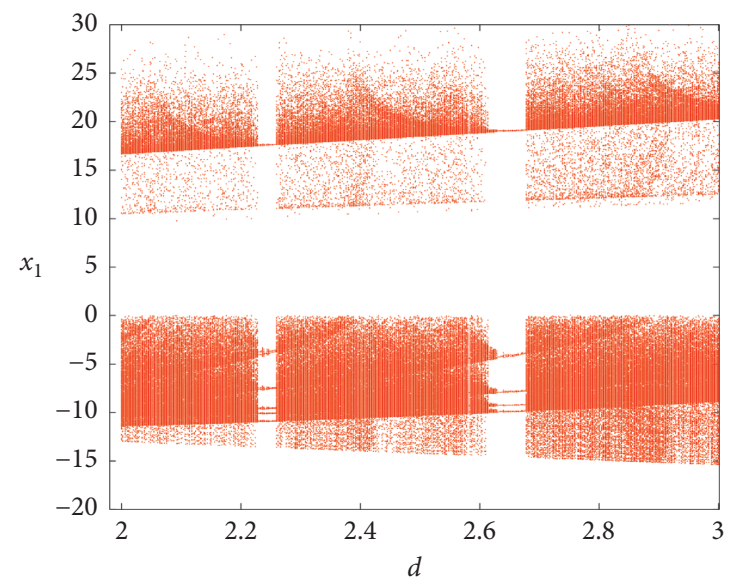

(a)

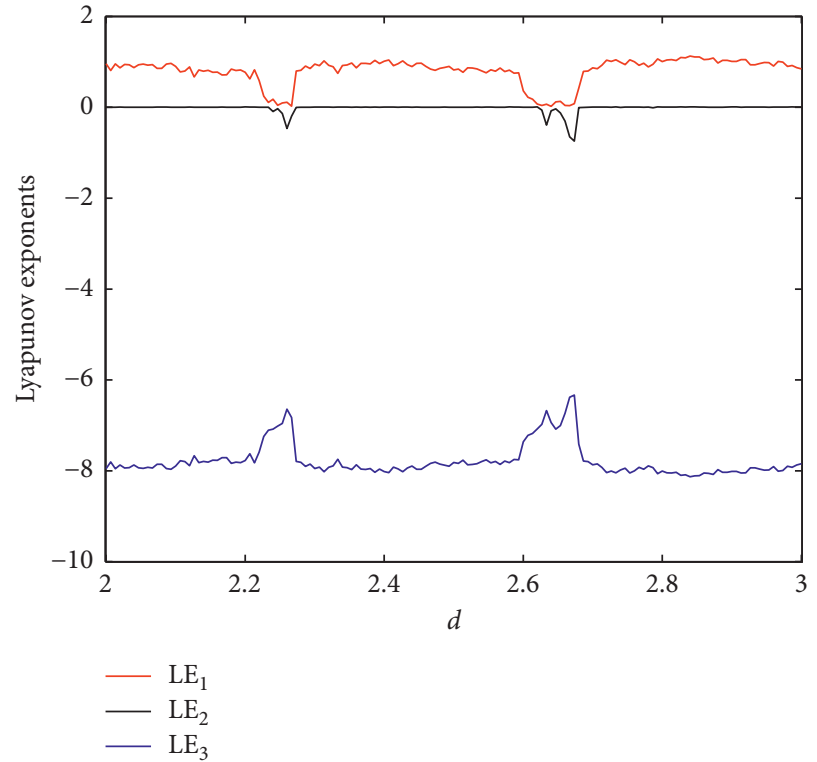

(b)

Figure 7: (a) Bifurcation diagram and (b) finite time Lyapunov exponent spectrum versus $d$.

$$
\begin{aligned}
P_{0}: \lambda_{1} & =-1, \lambda_{2}=14.2337, \lambda_{3}=-20.2337, \\
P_{1}: \lambda_{1} & =-9.7318, \lambda_{2}=1.3659+8.0449 i, \\
\lambda_{3} & =1.3659-8.0449 i, \\
P_{2}: \lambda_{1} & =-8.2073, \lambda_{2}=0.6036+12.5517 i, \\
\lambda_{3} & =0.6036-12.5517 i .
\end{aligned}
$$

Obviously, equilibrium point $P_{0}$ is a saddle-node with two-dimensional stable manifold and one-dimensional unstable manifold. And the equilibrium points $P_{1}$ and $P_{2}$ are saddle-focus points with two-dimensional unstable manifold and one-dimensional stable manifold.

The corresponding finite time Lyapunov exponents by orthogonal method are calculated as $0.926059,0.051479$, and -10.972296 . And the Kaplan-Yorke dimension is obtained as $D_{K Y}=2+(0.926059+0.051479) / 10.972296=2.0891$, revealing a fractional feature. Therefore, system (8) is chaotic. The typical chaotic phase diagrams and Poincare mapping on plane $x_{2}=0$ are depicted in Figure 5. 


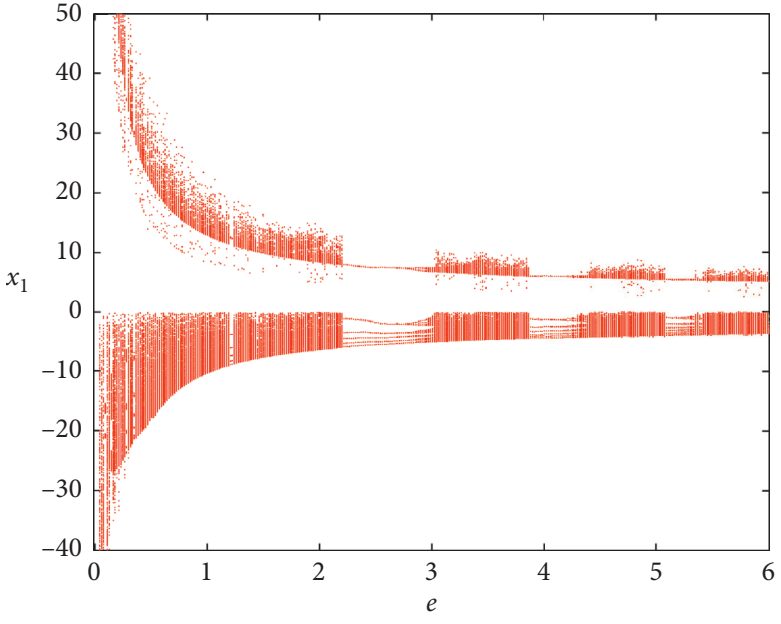

(a)

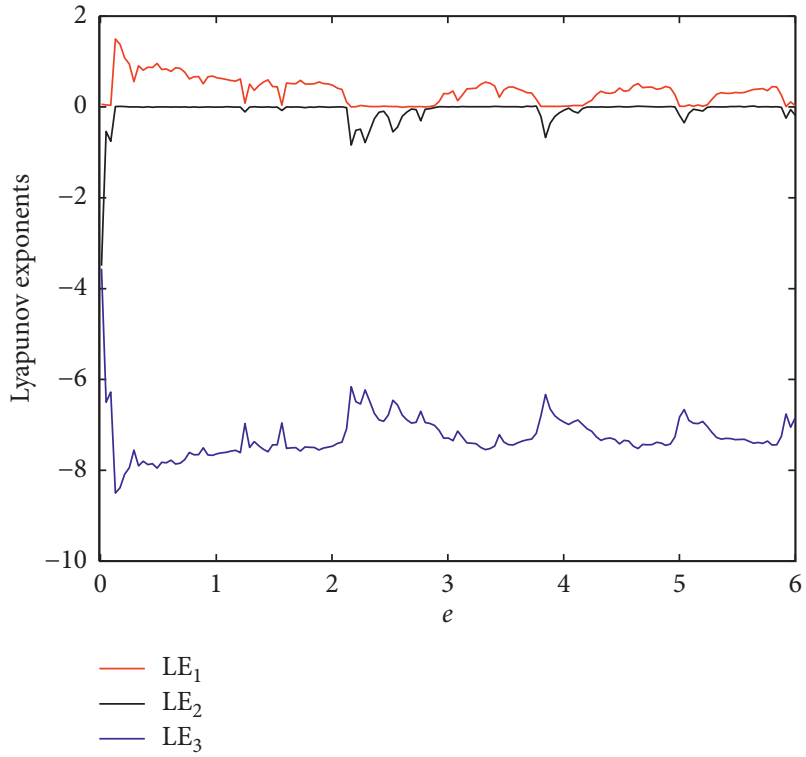

(b)

Figure 8: (a) Bifurcation diagram and (b) finite time Lyapunov exponent spectrum versus $e$.

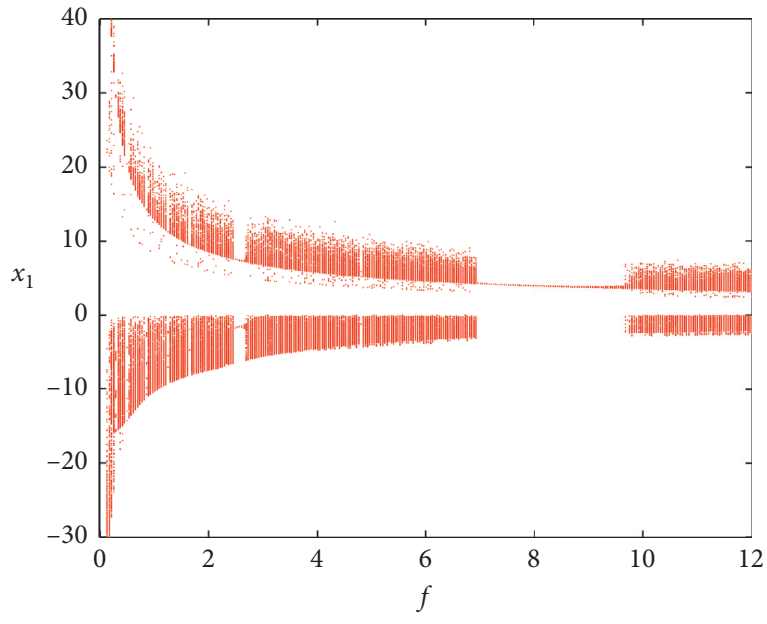

(a)

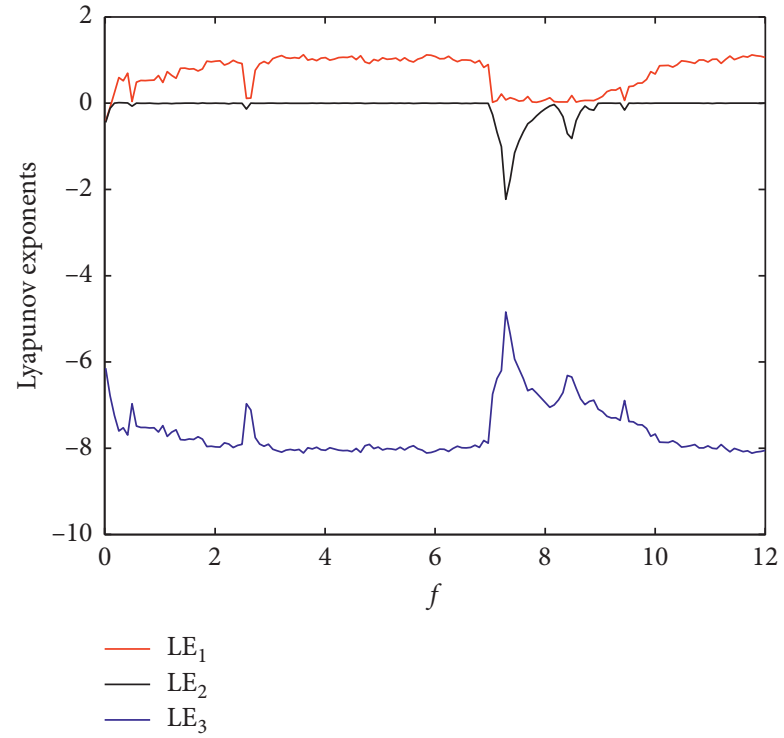

(b)

Figure 9: (a) Bifurcation diagram and (b) finite time Lyapunov exponent spectrum versus $f$.

3.2. Analysis of Phase Modulation. In system (8), coefficient $k$ is a phase parameter, which can control the signal phase of $x_{1}, x_{2}$ simultaneously. The phase reversal can be verified by the invariance of the transformation $\left(x_{1}, x_{2}\right.$, $\left.x_{3}, a, b, c, d, e, f, g, k\right) \longrightarrow\left(-x_{1},-x_{2}, x_{3}, a, b, c, d, e, f, g\right.$, $-k$ ). The bifurcation diagrams for $x_{1}$ and $x_{2}$ are reverse symmetrical, and the Lyapunov exponent spectrum is symmetrical about $k=0$, as depicted in Figure 6 . This further demonstrates that the sign of $k$ can control the polarity of $x_{1}$ and $x_{2}$, independent of the dynamics behavior.
3.3. Analysis of Amplitude Modulation. In the search for the property of amplitude modulation, it is surprising to find that for the presented system (8), there is no coefficient of nonlinearity employed to control the signal amplitude. The obtained result is different from the existing quadratic system [11-14] and the reported system (1). The most intuitive interpretation is the numerical simulations of bifurcation diagram and Lyapunov exponent spectrum versus the nonlinear parameter, as shown in Figures 7-9. Theoretically, according to the proposed method in [19], we cannot access appropriate variable 


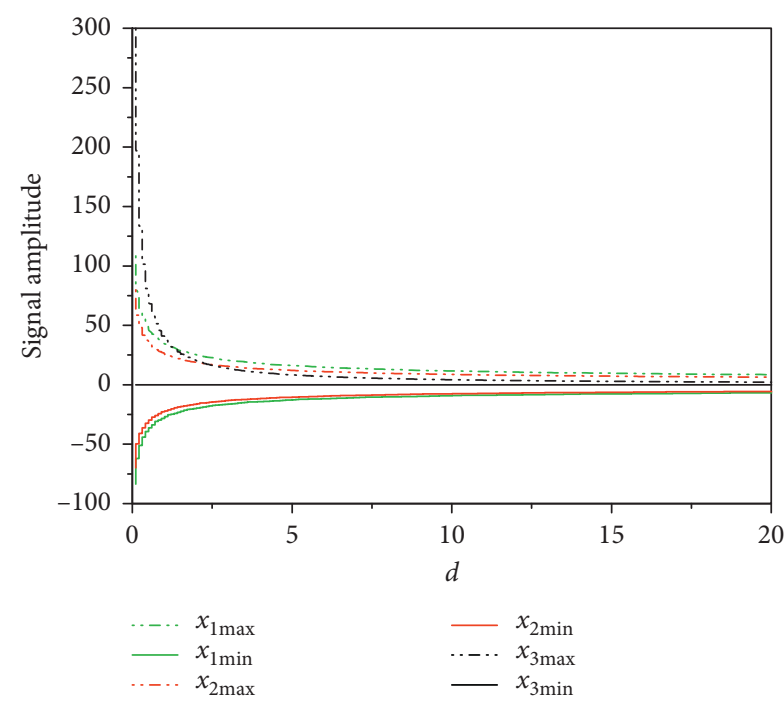

(a)

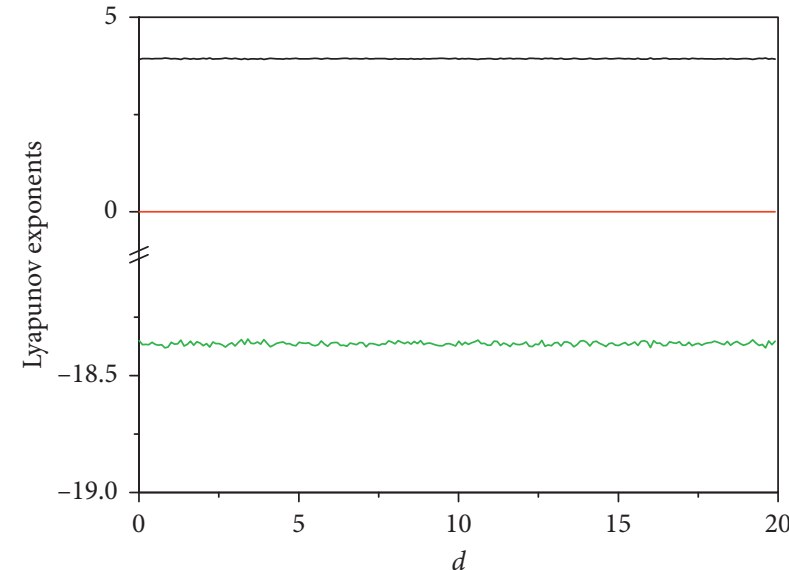

(b)

Figure 10: (a) Signal amplitude and (b) finite time Lyapunov exponent spectrum versus $d$.

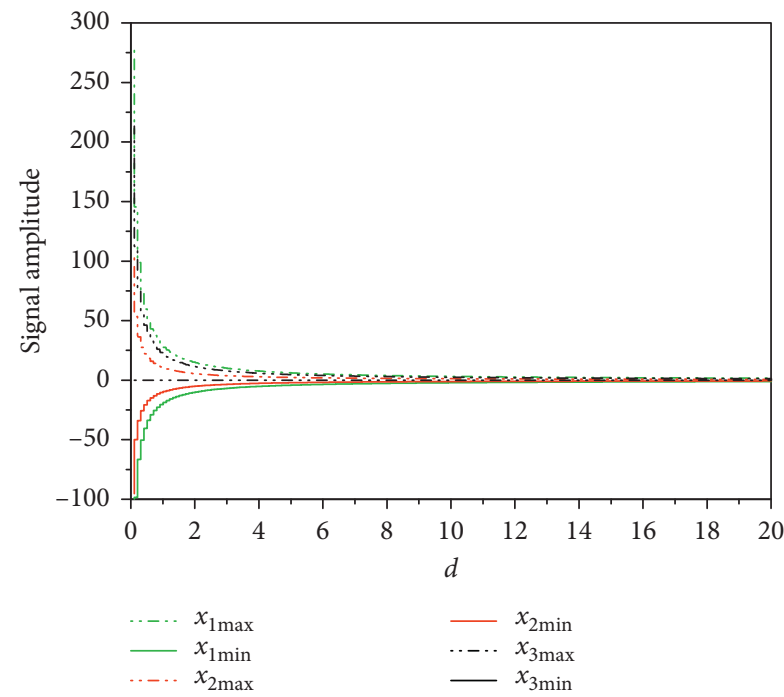

(a)

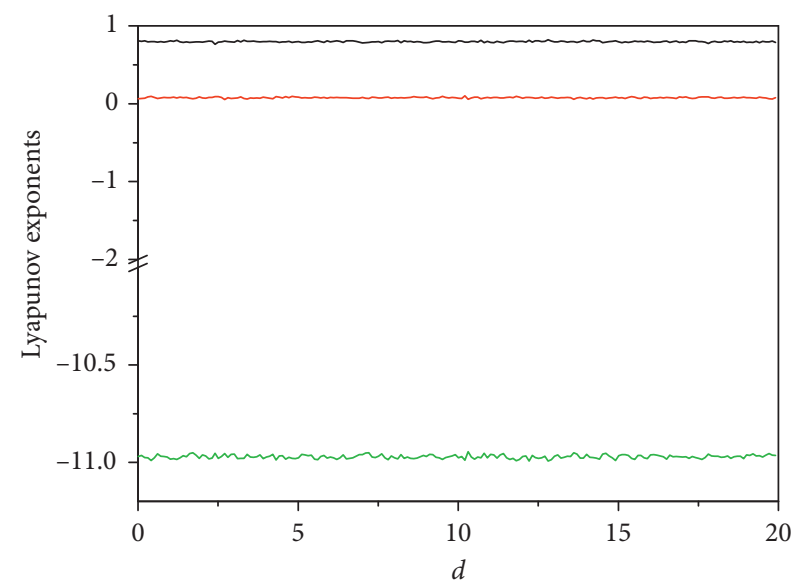

(b)

FIgURE 11: (a) Signal amplitude and (b) finite time Lyapunov exponent spectrum versus $d$.

substitution to realize the normalization of the system equation and characteristic equation [19].

\section{Amplitude Modulation Principle of Chaotic System}

As shown in the previous literatures [11-15], the coefficients of quadratic terms in smooth chaotic systems can modulate the signal amplitude of partial or total state variables. However, for systems (1) and (8) reported in this study, it is found that not all quadratic nonlinearity coefficients can be used to modulate the amplitude of signals. Therefore, when investigating dynamic properties of a chaotic system, we propose a naturally confusing but worthwhile question: "what is the possible principle for modulating the amplitude of chaotic signals?"

The physical significance of equilibrium point of dynamic system can be explained as zero velocity point. When the trajectory of a chaotic attractor is rescaled, the nonzero equilibrium point will deviate from the initial position. On the contrary, when the nonzero equilibrium point deviates from the initial position, the signal amplitude of the phase space trajectory can be rescaled. Therefore, the amplitude modulation principle of chaotic system with multiple equilibrium points can be described as follows: (1) in the mathematical representation of nonzero equilibrium point, the amplitude parameter is axisymmetric and (2) the location of the nonzero 


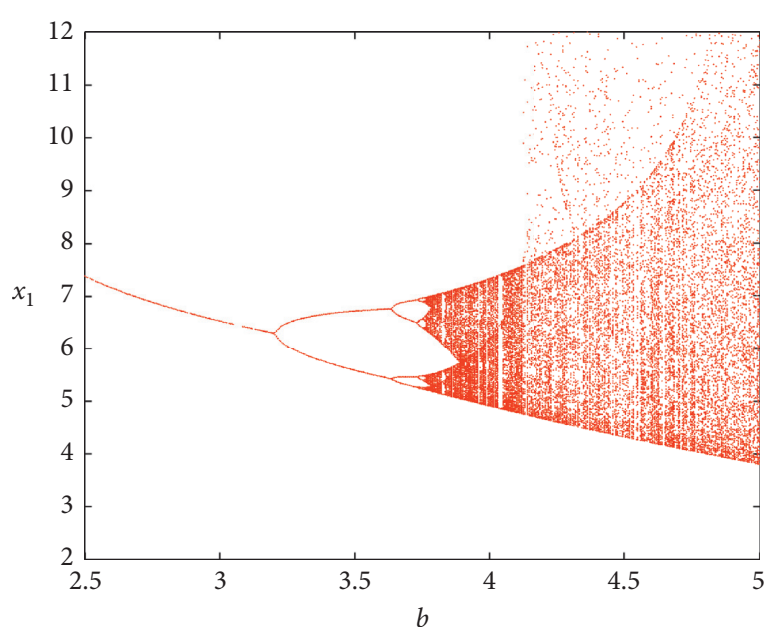

(a)

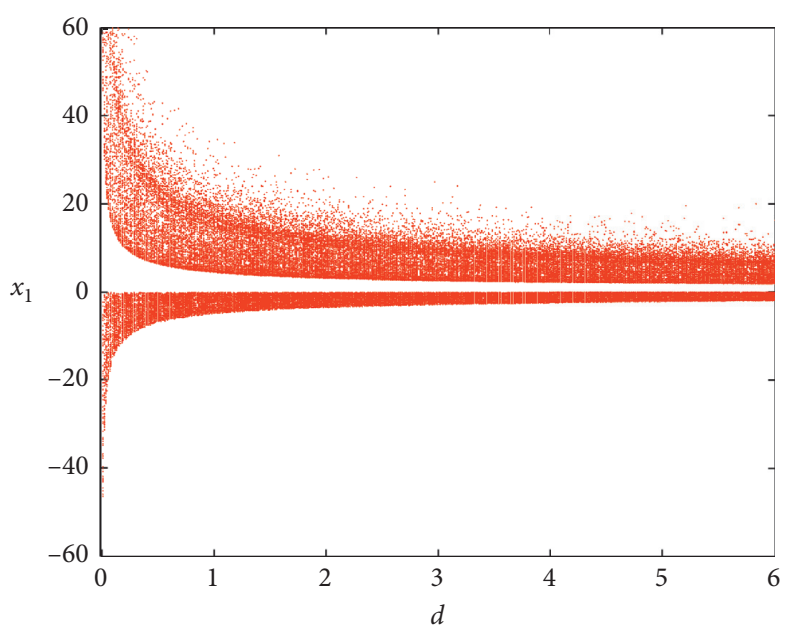

(c)

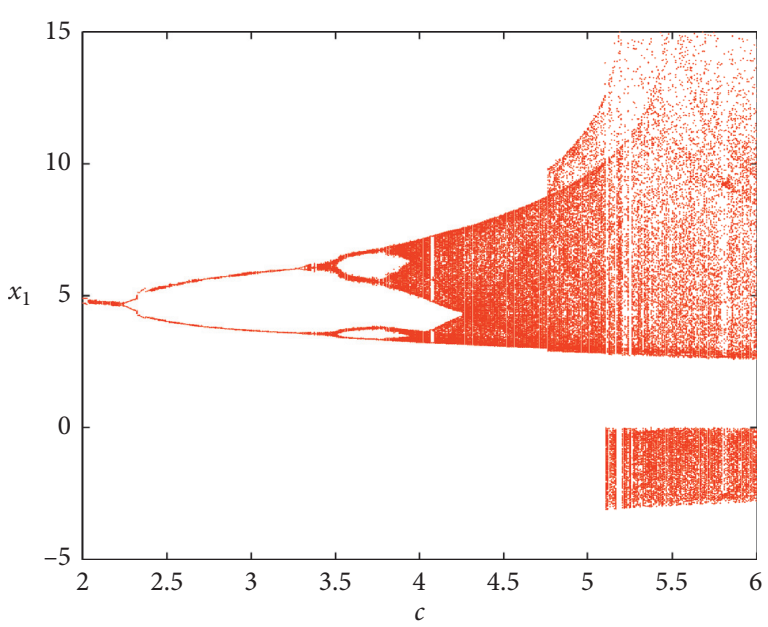

(b)

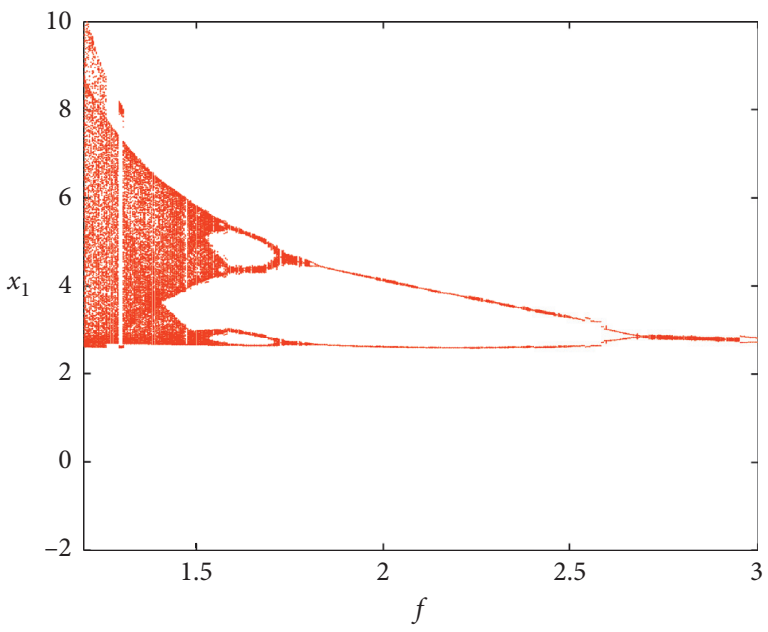

(d)

FIGURE 12: Modulation property of signal amplitude for system (15).

equilibrium point in phase space can be controlled by the amplitude parameter.

From the expression of nonzero equilibrium points of system (1), it can be seen that the parameters $d$ and $e$ of nonlinear term cannot modulate the location of equilibrium point $P_{1}, P_{2}, P_{3}$, or $P_{4}$. Accordingly, parameter $d$ or $e$ cannot modulate the signal amplitude. When we introduce a unified parameter $d$ in $x_{2} x_{3}$ and $x_{1} x_{3}$, system (1) is deduced to

$$
\left\{\begin{array}{l}
\dot{x}_{1}=a\left(x_{2}-x_{1}\right)+d x_{2} x_{3}, \\
\dot{x}_{2}=b x_{2}-d x_{1} x_{3}, \\
\dot{x}_{3}=-c x_{3}+f x_{1} x_{2},
\end{array}\right.
$$

and the four nonzero equilibrium points are

$$
\begin{aligned}
& P_{1}\left(\sqrt{\frac{b c}{d f}}, \frac{-a \sqrt{c}+\sqrt{c} \sqrt{a^{2}+4 a b}}{2 \sqrt{b d f}}, \frac{-a+\sqrt{a^{2}+4 a b}}{2 d}\right), \\
& P_{2}\left(\sqrt{\frac{b c}{d f}}, \frac{-a \sqrt{c}-\sqrt{c} \sqrt{a^{2}+4 a b}}{2 \sqrt{b d f}}, \frac{-a-\sqrt{a^{2}+4 a b}}{2 d}\right), \\
& P_{3}\left(-\sqrt{\frac{b c}{d f}}, \frac{a \sqrt{c}+\sqrt{c} \sqrt{a^{2}+4 a b}}{2 \sqrt{b d f}},-\frac{a+\sqrt{a^{2}+4 a b}}{2 d \sqrt{b f}}\right), \\
& P_{4}\left(-\sqrt{\frac{b c}{d f}}, \frac{a \sqrt{c}-\sqrt{c} \sqrt{a^{2}+4 a b}}{2 \sqrt{b d f}},-\frac{a-\sqrt{a^{2}+4 a b}}{2 d \sqrt{b f}}\right) .
\end{aligned}
$$


It is known that for equilibrium points $P_{1}$ and $P_{2}$, the set of symmetrical axis is $(0,-a \sqrt{c} / 2 \sqrt{b d f},-a / 2 d)$, and the set of symmetrical axis for equilibrium points $P_{3}$ and $P_{4}$ is $(0$, $a \sqrt{c} / 2 \sqrt{b d f},-a / 2 d \sqrt{b f})$. In addition, the parameter $d$ can modulate the location of the nonzero equilibrium points according to $\sqrt{1 / d}, \sqrt{1 / d}$, and $1 / d$, respectively. As a result, the parameter $d$ can modulate the amplitude of signal $x_{1}, x_{2}$, and $x_{3}$ according to $\sqrt{1 / d}, \sqrt{1 / d}$, and $1 / d$, respectively. The signal amplitude and Lyapunov exponent spectrum versus $d$ are depicted in Figure 10.

For the expression of nonzero equilibrium points of system (8), parameters $d, e$, and $f$ of quadratic terms cannot modulate the location of equilibrium point $P_{1}$ or $P_{2}$. Accordingly, parameter $d$, $e$, or $f$ cannot modulate the signal amplitude. To realize amplitude modulation in system (8), we introduce a unified parameter $d$ in each nonlinear term yielding

$$
\left\{\begin{array}{l}
\dot{x}_{1}=a x_{2}-g x_{1}+d x_{2} x_{3}, \\
\dot{x}_{2}=b x_{1}-d x_{1} x_{3}, \\
\dot{x}_{3}=k x_{1}-c x_{3}+d x_{1} x_{2},
\end{array}\right.
$$

and the two nonzero equilibrium points are deduced as

$$
\begin{aligned}
& P_{1}\left((a+b) \frac{-k+\sqrt{k^{2}+(4(b c g) /(a+b))}}{2 g d}, \frac{-k+\sqrt{k^{2}+(4(b c g) /(a+b))}}{2 d}, \frac{b}{d}\right), \\
& P_{2}\left((a+b) \frac{-k-\sqrt{k^{2}+(4(b c g) /(a+b))}}{2 g d}, \frac{-k-\sqrt{k^{2}+(4(b c g) /(a+b))}}{2 d}, \frac{b}{d}\right) .
\end{aligned}
$$

We know that for equilibrium points $P_{1}$ and $P_{2}$, the set of symmetrical axis is $(-k(a+b) / 2 g d,-k / 2 d, 0)$. In addition, the parameter $d$ can modulate the location of the nonzero equilibrium points according to $1 / d$, respectively. Similarly, parameter $d$ can modulate the amplitude of $x_{1}, x_{2}$, and $x_{3}$ according to $1 / d$, respectively. The signal amplitude and Lyapunov exponent spectrum versus $d$ are shown in Figure 11.

\section{Discussion and Conclusion}

Exploring the amplitude modulation phenomenon of chaotic signal is attractive yet recent topic of interest. This paper reported two chaotic systems with three quadratic cross-product terms and analyzed the property of amplitude modulation. By making an exhaustive study on the characteristics of nonzero equilibrium points, we attempt to address the possible principle for amplitude modulation. That is to say, the amplitude parameter in the expression of nonzero equilibrium point is symmetrical about some axis and can modulate the location of the nonzero equilibrium point in phase space.

The addressed principle can be popularized to highdimensional chaotic system and other chaotic systems except with quadratic nonlinearity [19-22], not relying on the type of nonzero equilibrium point. However, for the dynamical system with none, single, or an infinite number of equilibrium points [23-26], the principle is out of our consideration and deserves an in-depth study.

It must be reiterated that the proposed principle is just a prerequisite, but not a sufficient and necessary condition for amplitude modulation. As an interpretation, we consider the following system with cubic nonlinearity:

$$
\left\{\begin{array}{l}
\dot{x}_{1}=-a x_{1}+b x_{2} x_{3}, \\
\dot{x}_{2}=-c x_{2}^{3}+d x_{1} x_{3}, \\
\dot{x}_{3}=e x_{3}-f x_{1} x_{2} .
\end{array}\right.
$$

System (15) holds five equilibrium points, and the nonzero equilibrium points are described by

$$
\begin{aligned}
& P_{1}\left(\frac{e}{f} \sqrt{\frac{a c}{b d}}, \sqrt{\frac{a e}{b f}}, \frac{a}{b} \sqrt{\frac{c e}{d f}}\right), \\
& P_{2}\left(-\frac{e}{f} \sqrt{\frac{a c}{b d}}, \sqrt{\frac{a e}{b f}},-\frac{a}{b} \sqrt{\frac{c e}{d f}}\right), \\
& P_{3}\left(-\frac{e}{f} \sqrt{\frac{a c}{b d}},-\sqrt{\frac{a e}{b f}}, \frac{a}{b} \sqrt{\frac{c e}{d f}}\right), \\
& P_{4}\left(\frac{e}{f} \sqrt{\frac{a c}{b d}},-\sqrt{\frac{a e}{b f}},-\frac{a}{b} \sqrt{\frac{c e}{d f}}\right) .
\end{aligned}
$$

Clearly, the set of symmetrical axis for equilibrium points $P_{1}, P_{2}, P_{3}$, and $P_{4}$ is $(0,0,0)$. In addition, the parameter $b$ can modulate the location of the nonzero equilibrium points according to $\sqrt{1 / b}, \sqrt{1 / b}$, and $1 / b$; the parameter $c$ can modulate the location of the nonzero equilibrium points according to $\sqrt{c}, 1$, and $\sqrt{c}$; the parameter $d$ can modulate the location of the nonzero equilibrium points according to $\sqrt{1 / d}, 1$, and $\sqrt{1 / d}$; and the parameter $f$ can modulate the location of the nonzero equilibrium points according to $1 / f, \sqrt{1 / f}$, and $\sqrt{1 / f}$. However, it is found from the numerical experiment that only the nonlinear parameter $d$ can modulate the amplitude of $x_{1}, x_{2}$, and $x_{3}$ according to $\sqrt{1 / d}, 1$, and $\sqrt{1 / d}$, respectively, as plotted in Figure 12.

In spite of this, the principle provides a feasible mentality for analyzing amplitude modulation of chaotic signal, which can be briefly summarized from theoretical analysis to numerical confirmation. We hope that our work can 
constitute a stimulus and afford a subservient reference for further exploring the intrinsic amplitude modulation mechanism of chaotic systems.

\section{Data Availability}

The data used to support the findings of this study are included within the article.

\section{Conflicts of Interest}

The authors declare that there are no conflicts of interest regarding the publication of this paper.

\section{Acknowledgments}

This work was supported in part by the Hunan Provincial Natural Science Foundation of China (no. 2019JJ40109), the Research Foundation of Education Bureau of Hunan Province of China (nos. 18A314, 19C0864 and 16K037), Science and Technology Program of Hunan Province (nos. 2016TP1021 and 2019TP1014), the research and innovation project of the graduate students of Hunan Institute of technology (no. YCX2019A13), and the Science and Research Creative Team of Hunan Institute of Science and Technology (no. 2019-TD-10).

\section{References}

[1] M. Siewe, C. Tchawoua, and P. Woafo, "Melnikov chaos in a periodically driven Rayleigh-Duffing oscillator," Mechanics Research Communications, vol. 37, no. 4, pp. 363-368, 2010.

[2] A. Zarei, "Complex dynamics in a 5-D hyper-chaotic attractor with four-wing, one equilibrium and multiple chaotic attractors," Nonlinear Dynamics, vol. 81, no. 1-2, pp. 585-605, 2015.

[3] F. Peng, Q. Long, Z.-X. Lin, and M. Long, "A reversible watermarking for authenticating $2 \mathrm{D}$ CAD engineering graphics based on iterative embedding and virtual coordinates," Multimedia Tools and Applications, vol. 78, no. 19, pp. 26885-26905, 2019.

[4] E. Bilotta, F. Chiaravalloti, and P. Pantano, "Complexity and emergence of wave dynamics in a chain of sequentially interconnected Chua circuits," Mechanics Research Communications, vol. 68, pp. 9-17, 2015.

[5] C. Li, B. Feng, S. Li, J. Kurths, and G. Chen, "Dynamic analysis of digital chaotic maps via state-mapping networks," IEEE Transactions on Circuits and Systems I: Regular Papers, vol. 66, no. 6, pp. 2322-2335, 2019.

[6] Q. Zhao, C. Wang, and X. Zhang, "A universal emulator for memristor, memcapacitor, and meminductor and its chaotic circuit," Chaos, vol. 29, Article ID 013141, 2019.

[7] C. Li, K. Qian, S. He, H. Li, and W. Feng, "Dynamics and optimization control of a robust chaotic map," IEEE Access, vol. 7, pp. 160072-160081, 2019.

[8] A. Jimenez-Triana, G. Chen, and A. Gauthier, "A parameterperturbation method for chaos control to stabilizing UPOs," IEEE Transactions on Circuits and Systems II: Express Briefs, vol. 62, no. 4, pp. 407-411, 2015.

[9] X. Wang, J. Yu, C. Jin, H. H. C. Iu, and S. Yu, "Chaotic oscillator based on memcapacitor and meminductor," Nonlinear Dynamics, vol. 96, no. 1, pp. 161-173, 2019.

[10] Q. Lai, B. Norouzi, and F. Liu, "Dynamic analysis, circuit realization, control design and image encryption application of an extended Lü system with coexisting attractors," Chaos, Solitons \& Fractals, vol. 114, pp. 230-245, 2018.

[11] C. Li, L. Wu, H. Li, and Y. Tong, "A novel chaotic system and its topological horseshoe," Nonlinear Analysis: Modelling and Control, vol. 18, no. 1, pp. 66-77, 2013.

[12] C. Li, K. Su, and L. Wu, "Adaptive sliding mode control for synchronization of a fractional-order chaotic system," Journal of Computational and Nonlinear Dynamics, vol. 8, pp. 0310051-0310057, 2013.

[13] C. Li and J. Zhang, "Synchronisation of a fractional-order chaotic system using finite-time input-to-state stability," International Journal of Systems Science, vol. 47, no. 10, pp. 2440-2448, 2016.

[14] C. Li, Y. Tong, Y. Tong, and Y. Zeng, "Adaptive control and synchronization of a fractional-order chaotic system," Pramana, vol. 80, no. 4, pp. 583-592, 2013.

[15] X. Zhou, "A chaotic system with invariable Lyapunov exponent and its circuit simulation," Acta Physica Sinica, vol. 60, p. 100503, 2011.

[16] M.-S. Abdelouahab and N.-E. Hamri, "A new chaotic attractor from hybrid optical bistable system," Nonlinear Dynamics, vol. 67, no. 1, pp. 457-463, 2012.

[17] M.-F. Danca, N. Kuznetsov, and G. Chen, "Unusual dynamics and hidden attractors of the Rabinovich-Fabrikant system," Nonlinear Dynamics, vol. 88, no. 1, pp. 791-805, 2017.

[18] A. E. Matouk and H. N. Agiza, "Bifurcations, chaos and synchronization in ADVP circuit with parallel resistor," Journal of Mathematical Analysis and Applications, vol. 341, no. 1, pp. 259-269, 2008.

[19] C. Li, K. Su, and J. Zhang, "Amplitude control and projective synchronization of a dynamical system with exponential nonlinearity," Applied Mathematical Modelling, vol. 39, no. 18, pp. 5392-5398, 2015.

[20] Q. Yang and C. Chen, "A 5D hyperchaotic system with three positive Lyapunov exponents coined," International Journal of Bifurcation and Chaos, vol. 23, no. 6, p. 1350109, 2013.

[21] A. Sahab, M. Ziabari, and M. Modabbernia, "A novel fractional order hyperchaotic system with a quadratic exponential nonlinear term and its synchronization," Advances in Difference Equations, vol. 2012, no. 194, pp. 393-444, 2012.

[22] M.-F. Danca, "Attractors synthesis for a Lotka-Volterra like system," Applied Mathematics and Computation, vol. 216, no. 7, pp. 2107-2117, 2010.

[23] Z. Wei, "Dynamical behaviors of a chaotic system with no equilibria," Physics Letters A, vol. 376, no. 2, pp. 102-108, 2011.

[24] D. Cafagna and G. Grassi, "Chaos in a new fractional-order system without equilibrium points," Communications in Nonlinear Science and Numerical Simulation, vol. 19, no. 9, pp. 2919-2927, 2014.

[25] X. Wang and G. Chen, "A chaotic system with only one stable equilibrium," Communications in Nonlinear Science and Numerical Simulation, vol. 17, no. 3, pp. 1264-1272, 2012.

[26] P. Zhou and F. Yang, "Hyperchaos, chaos, and horseshoe in a $4 \mathrm{D}$ nonlinear system with an infinite number of equilibrium points," Nonlinear Dynamics, vol. 76, no. 1, pp. 473-480, 2014. 